Keio J. Med. 30: 133-139, 1981

\title{
POSTOPERATIVE LUMBAR CANAL STENOSIS DUE TO ANTERIOR SPINAL FUSION
}

\author{
KIYOSHI HIRABAYASHI, TETSUO MARUYAMA, KOICHI WAKANO, \\ KAMEO IKEDA and YOSHIAKI ISHII \\ Department of Orthopedic Surgery, School of Medicine, \\ Keio University, Tokyo, Japan
}

(Received for publication May 27, 1981)

\begin{abstract}
Three cases of postoperative lumbar spinal canal stenosis due to anterior spinal fusion were reported. In these cases stenosis occurred at the adjacent intervertebral disc level of the fused segment a considerable time after anterior spinal fusion performed for spondylolisthesis.

At the stenotic level of the lumbar spinal canal, hypertrophy of the lamina, body spur formation in the articular process, hypertrophy and calcification of the yellow ligament and narrowing of the lateral recess were noted.

These changes are believed to have been caused by excessive stress on the adjacent intervertebral disc, as is the case with stenosis occurring after posterior spinal fusion.

[Key words: postoperative lumbar spinal canal stenosis, anterior spinal body fusion, spondylolisthesis, intermittent claudication]
\end{abstract}

\section{INTRODUCTION}

There have been many reports ${ }^{1-5}$ concerning what is usually referred to as postoperative lumbar canal stenosis, which often occurs after laminectomy or posterior spinal fusion. We have recently experienced three cases in which stenosis occurred at the adjacent intervertebral disc level of the fused segment a considerable time after anterior spinal fusion had been performed for spondylolisthesis. In this report we present the three case histories and our considerations with regard to them.

\section{CASE REPORTS}

\section{Case 1}

A 56-year-old female visited our clinic with complaints of intermittent clau- 
dication and paresthesia of the both legs. At the age of 34 , low back pain occurred when carrying her child. At the age of 40 , we had diagnosed her as a case of degenerative spondylolisthesis (Fig. 1-a) and performed anterior spinal fusion at L.4/5. The postoperative course was uneventful and she was pain free for 14 years. In April 1975, however, after she had been caring for a sick person, low back pain recurred. By April 1977, paresthesia of both lower limbs also recurred and gradually worsened. By November of that year, she had to stop walking after 30 minutes because of the paresthesia and was rehospitalized.

On admission, a dulled sense of touch was observed in the L.5 nerve root area, but no Lasègue's sign was observed. Reflex and motor examination was normal.

$\mathrm{X}$-ray of the lumbar spine revealed pseudarthrosis of the L.4/5 space where the spinal fusion had been performed and narrowing of the disc of L.3/4, immediately above the fused area.

Myelography was performed between L.2 and 3, whereupon the contrast medium indicated a complete block at the L.3/4 immediately above due to extradural pressure (Fig. 1-b).

Discography was performed on L.3/4 and L.5/S.1. When performing this procedure on $\mathrm{L} .3 / 4$, the patient complained of low back pain during the injection of the contrast medium. According to discograms L.3/4 had degenerated severely and L.5/S.1 mildly.

Laminectomy was performed from L.3 to L.5. At the level of the lower half of the lamina of L.3, the posterior elements were compressing the dura mater posterolaterally and no epidural fatty tissue was observed. After the laminectomy, the dural tube was dilated posteriorally and pulsation was noted. Lumbar disc hernia was not observed. Posterolateral fusion was also performed on L.3/5 (Fig. 1-c).

It is now one year since the operation and all complaints have disappeared, which seems to indicate that the major cause of the patient's condition was lumbar canal stenosis at the level immediately above where anterior spinal fusion had been performed.

\section{Case 2}

A 64-year-old female visited our clinic with complaints of intermittent claudication and paresthesia of both lower limbs. Occasional low back pain started at the age of 42 , and pain in the left lower limb appeared at the age of 49. She was diagnosed as having a degenerative spondylolisthesis of L.3/4. We performed anterior spinal fusion at L.3/4 when the patient was 53 years old. The postoperative course was uneventful for eight years, but in 1972 she started to experience buttock pain when standing. In the spring of 1976, however, paresthesia of both lower limbs appeared and, although this complaint was 


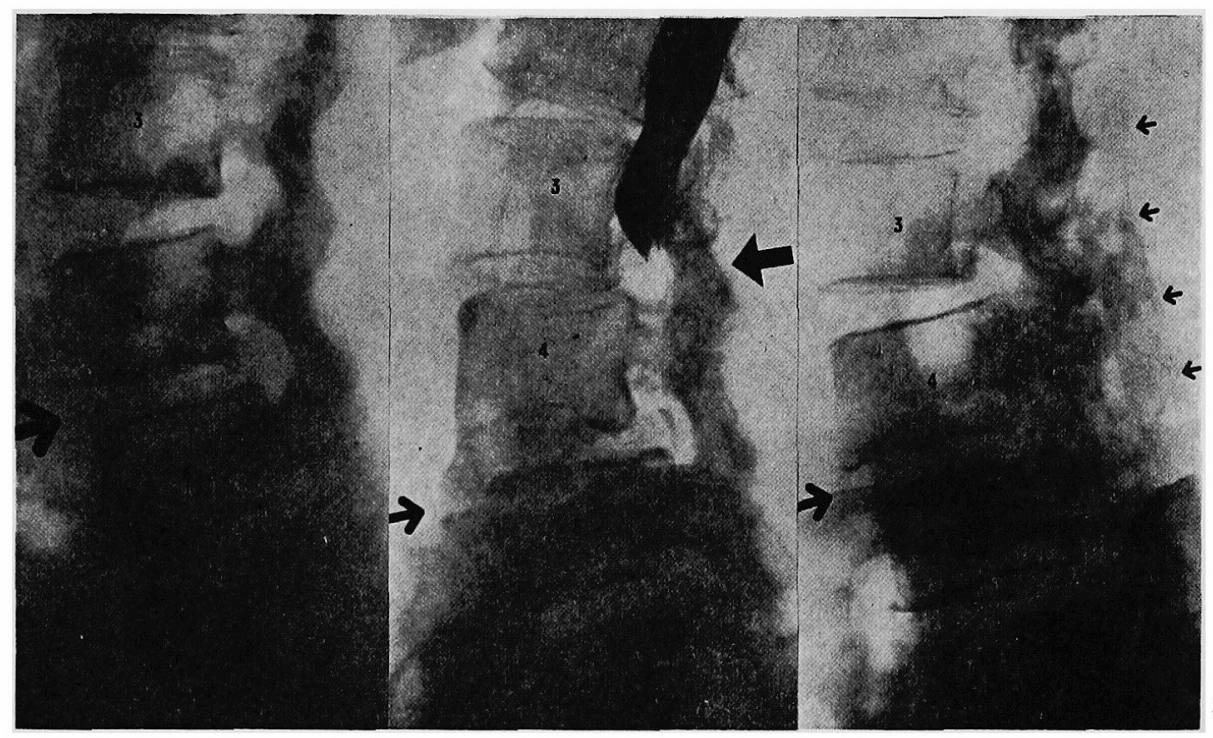

Fig. 1-a Pre-operative X-ray of case 1, 40-year-old female, showed degenerative (Nov. 1977) spondylolisthesis at the $\mathrm{L} .4 / 5$.

Fig. 1-b At the L.3/4, narrowing of the disc anterior slipping and complete block of the contrast medium with the redundant nerve roots was observed. At the L.4/5 Pseudarthrosis where anterior soinal fusion (Jan. 1979) was performed 13 years ago.

Fig. 1-c Postero-lateral fusion on the L.3/5 were completed. Pseudarthrosis of of the vertebral body at the L.4/5 (show in Fig. 1-b) already healed after laminectomy by posterolateral fusion on the L.3/5 performed one year and 3 months ago.

alleviated when the patient bent forward, she had to rest after walking 100 meters. Consequently, she was readmitted to our clinic in November of the same year.

On admission, with the exception of deficient Achilles tendon reflex, there were no neurological findings.

An X-ray check showed that there was osseous union of the L.3/4 where the anterior spinal fusion had been performed, but narrowing of the disc spaces immediately above and below the fused area was noted.

Myelography showed anterior and posterior pressure on L.3/4, where fusion had been performed, and complete block was observed below L.4/5 (Fig. 2).

Laminectomy was commenced from L.5 upwards to discover that the facet joints of L.4/5 were in a staircase formation and that the yellow ligament had hypertrophied with partial ossification. There was hardly any epidural fatty tissue and only after the laminectomy on L.3 was pulsation of the dural tube 


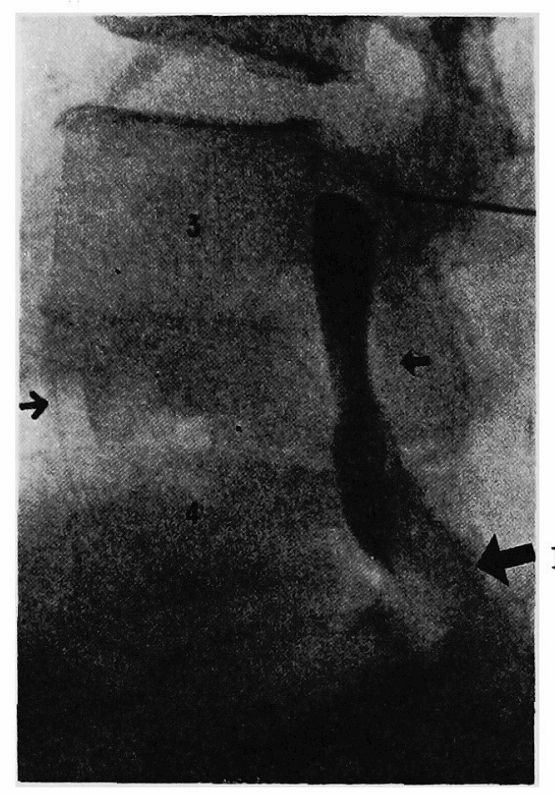

Fig. 2 Myelogram of case 2, 64-year-old female showed canal stenosis with anterior and posterior pressure on the L.3/4, where anterior spinal fusion was performed 11 years ago, and complete block of the contrast medium with narrowing of the disc space at the L.4/5.

noted. The nerve roots of L.4 and 5 were markedly strangulated at the lateral recess areas, which were expanded to remove the pressure. At L.4/5, disc protrusion was also observed and removed.

It is now three years since the operation and all complaints have improved, which seems to indicate that the major cause of the patient's condition was lumbar canal stenosis at the level of the articular process of L.4/5 immediately below where the anterior spinal fusion had been performed.

Case 3

A 76-year-old male visited our clinic with complaints of intermittent claudication. Paresthesia started to occur laterally in both legs at the age of 51 . Starting at the age of 55, dullness and discomfort of the lower limbs were noted when walking. After we diagnosed this patient as spondylolytic spondylolistheses, we performed anterior spinal infusion on L.5/S.1 in June 1961. The postoperative course was uneventful for nine years, but dullness of both legs recurred around 1970. A cold sensation from the buttocks down and intermittent claudication appeared in 1972. He was readmitted in 1974.

On admission, there were no physical findings indicating neurological disorders, and the X-ray check indicated that osseous union was complete after the fusion on L.5/S.1.

Myelography indicated canal stenosis at the L.4/5 level, immediately above where the fusion had been performed (Fig. 3).

Laminectomy was performed from L.3 to 5. Bony spur formation was observed around the intervertebral joints of L.3/4 and L.4/5 and hypertrophy 


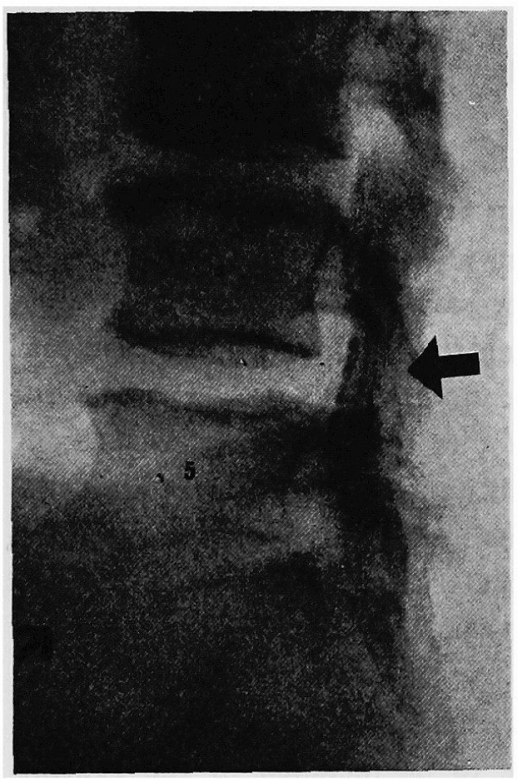

Fig. 3 Myelogram of case 3, 76-year-old male, showed canal stenosis with anterior and posterior compression at the L.4/5,. Immediately above where the anterior spinal fusion was performed at the L.5/S, 13 years ago.

of the yellow ligament and lamina was pressurizing the dural tube. No epidural fatty tissue was observed.

During the six years that have elapsed postoperatively, pain in the left buttock was noted when it was cold. In this case, it is suspected that we refer to as canal stenosis today was already present at time of the anterior spinal fusion, but that it did not become apparent for some time because of the anterior spinal fusion.

\section{DISCUSSION}

Laminectomy membrane, ligamental or osseous hypertrophy at the edge of the ablated periosteum, hypertrophy of the intervertebral joints, etc., are cited as pathological conditions of potoperative lumbar canal stenosis following laminectomy. Following posterior fusion, on the other hand, hypertrophy of the lamina at the level where the fusion was performed and protrusion of the intervertebral disc, hypertrophy of the yellow ligament or spur formation of the intervertebral joints at the level immediately above fused area, are cited as pathological condition., ${ }^{2,6,7}$ With three patients who underwent anterior spinal fusion, hypertrophy of the lamina was not observed at the level where the fusion had been performed, as is seen in patients who have undergone posterior spinal fusion. This indicates that vertebral body fusion is more reliable from the standpoint of dynamics than posterior fusion. 
However, hypertrophy of the lamina, body spur formation in the articular process, hypertrophy and calcification of the yellow ligament, narrowing of the lateral recess, lack of epidural fatty tissue, etc., were noted at the level immediately above or below where anterior fusion had been performed. These changes are believed to have been causd by excessive stress on the adjacent intervertebral disc, as is the case with stenosis occurring after posterior spinal fusion., ${ }^{8,9}$

Consequently, it can be said that it is necessary to consider the possible adverse effects on the adjacent discs when deciding the level at which to perform vertebral body fusion. Disc degenerations are caused by not only pathological conditions, but physical aging. When the severe disc degenerations are present at the adjacent levels, physically or pathologically, unstable spine or postoperative stenosis will occur more frequently as adverse effect on the adjacent discs following spinal fusion. Therefore, anterior spinal fusion should be performed on the degenerated adjacent disc space simultaneously as relative indication of spinal fusion. This is the reason why discography is included in the routine preoperative examination for lumbar disc lesions. The cases presented here occurred before the era when discography came to be included in the routine examinations for lumbar disc lesions. In view of this, we suspect that multivertebral fusion was indicated initially. Although we believe that we have paid sufficient attention since then to the selection of the intervertebral disc level to be anteriorly fused depending on the patient's age and the degree of degeneration of the discs, we plan to undertake a long-term study of the postoperative course of the patients of whom we have performed anterior spinal fusion.

In these three cases with postoperative lumbar canal stenosis, there are no additional notable findings with regard to the diagnosis and treatment. In fact, we have obtained favorable results with a wide range of laminectomies in these cases.

\section{REFERENCES}

1. Arct, W. A.: Die Wirbelkanalstenose nach Spondylodese. Arch. Orthop. u. Unfallchir. 83: 353-364, 1975

2. Broadsky, A. E.: Post-laminectomy and post-fusion stenosis of the lumbar spine. Clin, Orthop. 115: 130-139, 1976

3. Echizenya, T. and Kaneda, K.: Postoperative lumbar spinal canal stenosis. Clin. Orthop. (Jpn) 11: 729-732, 1976 (in Japanese)

4. Nishioeda, M. and Kataoka, O.: Postoperative lumbar spinal stenosis. Central JaJp. J. Orthop. Traumat. 21: 108-111, 1978 (in Japanese)

5. Quencer, R. M., Murtagh, F. R., Post, M. J. D., et al.: Postoperative bony stenosis of the lumbar spinal canal: evaluation of 164 symptomatic patients with axial radiography. Amer. J. Roent. 131: 1059-1064, 1978

6. Kirkaldy-Wilisl, W. H., Wedge, J.H., et al.: Pathology and pathogenesis of lumbar spondylosis and stenoses. Spine. 3: 319-328, 1978

7. Watanabe, R. and Akaboshi, Y.: Postoperative Wirbelkanalstenose, Z. Orthop. 
115: $386-391,1977$

8. Hamano, Y.: The influence of vertebral body fusion on the adjacent intervertebral discs-an experimental study. J. Jap. Orthop. Ass. 42: 1088-1102, 1968 (in Japanese)

9. Yamaguchi, M.: Photoelastic study on influence of spine fusion upon the adjacent vertebrae. Arch. Jap. Chir. 35: 955-980, 1966 